\title{
Stabilizing chaos in a dynamic macroeconomic model
}

\author{
Leo Kaas ${ }^{*}$ \\ Department of Economics, University of Bielefeld, P.O.Box 100131, D-3350I Bielefeld, Germany
}

\begin{abstract}
Within a macroeconomic disequilibrium model it turns out that stationary and simple adaptive policies are not capable of stabilizing efficient steady states and lead to periodic or irregular fluctuations for large sets of policy parameters. The application of recent control methods for chaotic dynamical systems shows that the government can, in principle, stabilize an unstable Walrasian equilibrium in a short time by varying income tax rates or government expenditures. Different stabilizing policies are analyzed and compared with regard to their efficiency.
\end{abstract}

JEL classification: E32; E62

Keywords: Disequilibrium models; Nonlinear dynamics; Stabilization policy; Controlling chaos

\section{Introduction}

In a simple dynamic multiplier-accelerator model Phillips (1954) showed that very intuitive stabilization policies can lead throughout to undesired oscillations and instabilities, and that only certain choices of policy rules will really damp the oscillations. In the 1960s and 1970s applications of optimal control theory to models of economic growth and to short-term econometric models have been studied intensively. These approaches mostly worked with linear models. Yet there exist good arguments to prefer nonlinear modeling. Nonlinearities occur automatically in micro-founded macroeconomic models, and should be incorporated explicitly when a description of the dynamics away from the steady state is desired. They are also capable of explaining stationary, cyclical and irregular dynamic behavior cndogenously. However, in nonlinear models it might be the case that regular motion need not be preferred to irregular motion,

* Corresponding author. e-mail: LKAAS@WIWI.UNI-BIELEFELD.DE 
since appropriate welfare measures take on worse values on regular than on irregular time paths.

In this paper a nonlinear dynamic macroeconomic model is introduced which displays such a trade-off between regularity and efficiency. Its short-run structure is related to Neo-Keynesian models developed by Benassy (1975) and Malinvaud (1977) who built on the seminal work of Clower (1965). Models of this kind describe the emergence of different disequilibrium regimes due to rationing of supply and demand at prevailing prices and wages, and are useful to understand phenomena such as unemployment and inflation in a sound micro-founded framework. In their original form they lacked an explanation of why prices are not at their market clearing level. Following Böhm (1993), price and wage adjustments are introduced explicitly based on the 'law of supply and demand'. They could be combined with expectation augmentations or cost push terms, but this is a task for another place.

It turns out that a Walrasian equilibrium steady state only exists at a balanced government budget. The government, however, cannot stabilize such a state by stationary policies. Stationarity can only be achieved at disequilibrium states of high unemployment or high inflation, while policies which succeed in the short run lead eventually to periodic or irregular fluctuations. In particular, balanced budget policy rules do not stabilize a Walrasian steady state.

Similar to Day's (1994) result the model shows that policies leading to fluctuations may exhibit better average performance than policies with inefficient steady states. It is, however, desirable to know if policies exist which can achieve a regular and efficient dynamics. Such policies can be found by the application of recent control techniques for chaotic dynamical systems. One is a local control method (Ott et al., 1990) which needs knowledge of first-order coefficients at the steady state but which may exhibit very long transients. This problem can be overcome by a global targeting method due to Shinbrot et al. (1990) which makes use of the sensitive dependence on initial conditions. The application of these methods defines tax rate and expenditure policies that stabilize the Walrasian equilibrium in a very short time. Since it is not behaviorally plausible that the government has complete knowledge of the underlying dynamic system, it is necessary to study whether these policies can be applied under incomplete information of the dynamics. In particular, it will be investigated whether these policies display correlations with observable market signals and could thus be implemented in spite of ignorance of the dynamic law of motion.

After introducing the model in the next section, Section 3 deals with the comparative dynamics of different policies. Section 4 introduces the control methods for chaotic systems which are applied in Section 5 to obtain stabilizing policies. Four different policies are compared with regard to their efficiency which can be measured by the time needed to reach the equilibrium.

\section{A macroeconomic disequilibrium model}

Consider a dynamic Neo-Keynesian model in the tradition of Benassy (1975), Malinvaud (1977). Its short-run supply and demand structure is derived from micro- 
economic behavioral assumptions, while temporary fixed prices and wages allow disequilibrium allocations due to the possibility of rationing in different markets. Prices and wages adjust from one period to the next according to disequilibrium signals, while the money balances dynamics results from the savings behavior of households and the policy. This defines a dynamical system with two state variables, the real wage and the real money balance. A more detailed presentation is given in Böhm (1993),Böhm et al. (1994).

The economy consists of a young and an old household, a firm, the government, and markets for a consumption good and labor. In the beginning of every period $t$ the goods price $p_{t}$, the nominal wage $w_{t}$ and the nominal money stock $M_{t}$, which is held by old households only, are given. The agents are described by the following behavioral assumptions.

The households: Households are assumed to live for two periods. Young households supply a fixed amount of labor $L_{\max }$ and receive labor as well as profit income. In both periods of their life they demand the consumption good, and the difference between income and consumption is transferred to the next period as savings in the form of money. Thus, at the beginning of period $t$ the old household holds the nominal money stock $M_{t}$, which leads to his notional demand for the consumption good $m_{r}:=M_{r} / p_{r}$. Young households' propensity to consume out of net income is given by a constant $\bar{c} \in(0,1)$.' Thus, the desired consumption of both households in period $t$ for a real net income of young houscholds $y_{t}^{\text {net }}$ is given by $x_{t}^{*}=m_{t}+\bar{c} \cdot y_{t}^{\text {net }}$.

The firm: The firm produces the consumption good with labor as the only variable input factor. Investment or inventory holding is excluded. The production technology is described by a neoclassical production function of the form $y=F(L)=\frac{a}{b} L^{b}$ with $a>0$ and $b \in(0,1)$. Profit maximization at the given real wage $\alpha_{t}:=w_{t} / p_{t}$ leads to the notional demand for labor $L^{*}\left(\alpha_{t}\right)$ and the notional supply of goods $y^{*}\left(\alpha_{t}\right)=F\left(L^{*}\left(\alpha_{t}\right)\right)$.

The government: The government levies a proportional income tax rate $\tau \in[0,1]$ on young household's income and demands a quantity $g \geq 0$ of the consumption good. Eventual budget deficits resp. surpluses will be financed via money creation resp. destruction. Thus, the change in the money stock $\Delta M_{t}=M_{t+1}-M_{t}$, that is, ex post net savings of the household sector, equals the budget deficit/surplus of the government.

The unique feasible allocation in period $t$ at given real wage $\alpha_{t}$, real balance $m_{t}$ and policy parameters $(g, \tau)$ is defined by a pair $\left(y_{t}, L_{t}\right)$ of production and employment, such that exchanges on the goods and labor markets take place at the minimum of effective supply and demand after taking into account eventual spill-overs from the other market. This leads to the following determination of $\left(y_{t}, L_{t}\right)$ : Let $y^{D}\left(m_{i} ; g, \tau\right)$ denote the incomeconsistent aggregate effective consumption demand, that is, the solution of

$$
y^{D}=m_{t}+g+\bar{c} \cdot(1-\tau) \cdot y^{D},
$$

\footnotetext{
1 This can be derived from a Cobb-Douglas utility function between today's and tomorrow's consumption. If a general homothetic function is used, expectations of future prices have to be taken into account (Cf. Böhm, 1993).
} 
which is given by

$$
y^{D}\left(m_{t} ; g, \tau\right)=\frac{m_{t}+g}{1-\bar{c} \cdot(1-\tau)} .
$$

The cffective goods supply is determined by $y^{S}\left(\alpha_{t}\right):=\min \left(y^{*}\left(\alpha_{t}\right), F\left(L_{\max }\right)\right)$. Thus the unique feasible allocation is determined by

$$
y_{t}=\mathscr{Y}\left(\alpha_{t}, m_{t} ; g, \tau\right):=\min \left(y^{D}\left(m_{t} ; g, \tau\right), y^{S}\left(\alpha_{t}\right)\right)
$$

and

$$
L_{t}=\mathscr{L}\left(\alpha_{t}, m_{t} ; g, \tau\right):=F^{-1}\left(\mathscr{Y}\left(\alpha_{t}, m_{t} ; g, \tau\right)\right)
$$

Feasible allocations are distinguished with regard to their rationing situations. In particular, the so-called disequilibrium regimes are defined by separation of the state/ parameter space $\left(\alpha_{t}, m_{i} ; g, \tau\right) \in \mathbb{R}_{++}^{2} \times \mathbb{P}_{+} \times[0,1]$ into the following open subsets:

1. K (Keynesian unemployment): Supply rationing on both markets, that is, $y_{t}=$ $y^{D}\left(m_{t} ; g, \tau\right)<y^{*}\left(\alpha_{t}\right)$ and $L_{t}=F^{-1}\left(y^{D}\left(m_{t} ; g, \tau\right)\right)<L_{\max }$.

2. C (Classical unemployment): Demand rationing on the goods market and supply rationing on the labor market, that is, $y_{t}=y^{*}\left(\alpha_{t}\right)<y^{D}\left(m_{t} ; g, \tau\right)$ and $L_{t}<L_{\max }$.

3. I (Repressed inflation): Demand rationing on both markets, that is, $y_{t}=F\left(L_{\max }\right)<$ $y^{D}\left(m_{t} ; g, \tau\right)$ and $L_{t}=L_{\max }<L^{*}\left(\alpha_{t}\right)$.

The case of supply rationing on the goods market and demand rationing on the labor market $\mathbf{U}$ (Underconsumption) degenerates to the boundary $\overline{\mathbf{K}} \cap \overline{\mathbf{I}}$. Furthermore, there are three other boundary cases: Two with one market cleared $(\overline{\mathbf{K}} \cap \overline{\mathbf{C}}$ and $\overline{\mathbf{C}} \cap \overline{\mathbf{I}})$, and the Walrasian equilibrium $\mathbf{W}=\overline{\mathbf{K}} \cap \overline{\mathbf{I}} \cap \overline{\mathbf{C}}$, which is the only state with realization of all notional demands and supplies.

The dynamical equations: In order to define the dynamics of the money stock, we have to specify the rationing scheme on the goods market. If there is demand rationing on the goods market, it is assumed that the young household is rationed first, the old household second and that the government is rationed only if both households are rationed to zero. Furthermore, it is assumed that not consumed savings of a demandrationed old household will be paid to the government. The rationing scheme implies that actual consumption of the young household is $x_{t}=\max \left(0, y_{t}-m_{t}-g\right)$. Since the money stock in period $t+1$ is equal to the savings of the young household in period $t$, it follows

$$
M_{t+1}=(1-\tau) p_{t} y_{t}-p_{t} x_{t}=p_{t}\left(\min \left(y_{t}, g+m_{t}\right)-\tau y_{t}\right)
$$

Prices and wages are assumed to change according to the so-called 'law of supply and demand,' that is, prices increase (resp. decrease) if and only if there is an excess demand (resp. supply). This can be written as

$$
p_{t+1}=p_{t}\left(1+\mathscr{P}\left(\alpha_{t}, m_{i} ; g, \tau\right)\right) \quad \text { and } \quad w_{t+1}=w_{t}\left(1+\mathscr{W}\left(\alpha_{t}, m_{t} ; g, \tau\right)\right)
$$


where the functional specifications of the growth rates are

$$
\begin{aligned}
& \mathscr{P}(\cdot):= \begin{cases}\mathscr{P}_{\max } \frac{y^{D}\left(m_{t} ;, \tau\right)-\mathscr{g}(\cdot)}{y^{D}\left(m_{t} ; g, \tau\right)} & \text { if } y^{D}\left(m_{t} ; g, \tau\right)>\mathscr{Y}(\cdot), \\
\mathscr{P}_{\min } \frac{\mathscr{P}(\cdot)-y^{*}\left(\alpha_{t}\right)}{y^{*}\left(\alpha_{t}\right)} & \text { otherwise, }\end{cases} \\
& \mathscr{W}(\cdot):= \begin{cases}\mathscr{W}_{\max } \frac{L^{*}\left(\alpha_{t}\right)-\mathscr{L}(\cdot)}{L^{*}\left(\alpha_{t}\right)} & \text { if } L^{*}\left(\alpha_{t}\right)>\mathscr{L}(\cdot), \\
\mathscr{W}_{\min } \frac{\mathscr{L}(\cdot)-L_{\max }}{L_{\max }} & \text { otherwise. }\end{cases}
\end{aligned}
$$

Here ( $)$ stands for the tuple $\left(\alpha_{t}, m_{t} ; g, \tau\right)$ and $\mathscr{P}_{\min }, \mathscr{P}_{\max }, \mathscr{W}_{\min }, \mathscr{W}_{\max } \in[0,1]$ denote the flexibilities of prices and wages to below and to above. It is easy to see that the functions $\mathscr{P}$ and $\mathscr{W}$ depend piecewise differentiable on $\left(\alpha_{t}, m_{t} ; g, \tau\right)$ in every disequilibrium regime $\mathbf{K}, \mathbf{C}$ and $\mathbf{I}$. The complete dynamical system can be written as

$$
\mathscr{F}(\cdot ; g, \tau): \mathbb{R}_{++}^{2} \rightarrow \mathbb{R}_{++}^{2}, \quad\left(\alpha_{t}, m_{t}\right) \mapsto\left(\alpha_{t+1}, m_{t+1}\right)
$$

with

$$
\begin{aligned}
\alpha_{t+1} & =\alpha_{t} \frac{1+\mathscr{W}\left(\alpha_{t}, m_{t} ; g, \tau\right)}{1+\mathscr{P}\left(\alpha_{t}, m_{t} ; g, \tau\right)} \\
m_{t+1} & =\frac{1}{1+\mathscr{P}\left(\alpha_{t}, m_{t} ; g, \tau\right)}\left(\min \left(\mathscr{Y}\left(\alpha_{t}, m_{t} ; g, \tau\right), m_{t}+g\right)-\tau \mathscr{Y}\left(\alpha_{t}, m_{t} ; g, \tau\right)\right) .
\end{aligned}
$$

In Böhm et al. (1994) it was shown that this dynamical system exhibits various forms of dynamic behavior, like stationarity or cyclicity, coexisting cycles with complicated basins of attraction and chaotic limit behavior. The latter point was supported by the numerical detection of a Smale's horseshoe.

\section{The role of policy parameters}

We will first concentrate on properties of stationary states at given stationary policies. Detailed proofs of the following Propositions are contained in Kaas (1995).

First, it should be noted that a Classical state can never be stationary, since wages have to fall and prices have to rise because of an excess demand (resp. supply) on the goods (resp. labor) market, and therefore real wages fall in the Classical regime. Furthermore, the disequilibrium type of the steady state depends only on the sign of the government budget at full employment. For example, a budget deficit implies an increasing money stock, which can be compatible with a stationary real balance if prices rise with the same growth rate. To keep real wages constant, the nominal wages have also to increase. Thus, a steady state requires general excess demand and must therefore be contained in $\mathbf{I}$. A similar argument shows that a full employment government surplus requires a Keynesian (i.e. excess supply) steady state.

To prove the existence of a steady state, we have to make certain assumptions on price and wage flexibilities. For instance, if wages are not sufficiently flexible, a high budget deficit implies a high price inflation at the stationary real balance, which cannot be matched by a wage inflation on the same level. Thus, in this case real wages cannot be 
stationary. In the Keynesian case we have to claim that prices are flexible enough to below. The results are summarized in the next proposition.

\section{Proposition 1:}

1. If $g<\tau F\left(L_{\max }\right)$ and $\mathscr{P}_{\min }$ is not too small, then there exists a steady state in $\mathbf{K}$.

2. If $g>\tau F\left(L_{\max }\right)$ and $\mathscr{W}_{\max }$ is not too small, then there exists a steady state in $\mathbf{l}$.

3. If $g=\tau F\left(L_{\max }\right)$, then $\mathbf{W}$ is a steady state.

4. There is at most one steady state of $\mathscr{F}(\cdot ; g, \tau)$ for each $(g, \tau)$.

Since $\mathscr{F}$ is non-differentiable in a Walrasian steady state, a local stability analysis can be carried out only for disequilibrium steady states. The result is contained in the next Proposition, which follows from eigenvalue calculations.

\section{Proposition 2:}

Any steady state $(\bar{\alpha}, \bar{m} ; g, \tau) \in \mathbf{K} \cup \mathbf{I}$ can lose its local stability only via a period doubling bifurcation. If it is unstable, one eigenvalue still has a modulus less than one. Moreover, if all other parameters are kept constant, then $(\bar{\alpha}, \bar{m} ; g, \tau) \in \mathbf{I}$ can lose local stability via a decrease of $g$ or an increase of $\tau$, while $(\bar{\alpha}, \bar{m} ; g, \tau) \in \mathbf{K}$ can lose local stability via an increase of $g$ or a decrease of $\tau$.

This result implies that a well-intended fiscal policy can destabilize the steady state and therefore become ineffective in the long run. In fact, even though an expansive fiscal policy under Keynesian unemployment lowers excess supply in the steady state, its longterm consequences are ambiguous. For Inflationary steady states the same holds true: Although a contractive fiscal policy lowers the short-term inflation rate and excess demand, it may destabilize the steady state. All this makes clear that one has to be cautious in adopting 'intuitive' results from comparative statics to a dynamic framework. ${ }^{2}$

Choosing the parameters equal to

$$
a=1, b=0.9, L_{\max }=1, \bar{c}=0.5, \mathscr{P}_{\min }=\mathscr{P}_{\max }=\mathscr{W}_{\min }=\mathscr{W}_{\max }=0.6,
$$

we plotted the Inflationary (resp. Keynesian) bifurcation curves in $(g, \tau)$-parameter space in Fig. 1. The steady state is stable for shaded parameter combinations.

For those policy mixes which allow a stationary Walrasian equilibrium the steady state is unstable. In addition, a 2-parameter bifurcation shows that for those parameter combinations only higher cyclicity or irregular behavior can occur. The picture suggests that regularity can only be achieved at the cost of a strong disequilibrium state and that it is not possible to stabilize a desirable state by a stationary policy $\mathrm{mix}^{3}{ }^{3}$

One might expect that, in contrast to the negative results for stationary policies, certain plausible policy rules stabilize the Walrasian equilibrium. We investigated this for the case of a pay-as-you-go, balanced budget policy, where the government spends in period

\footnotetext{
2 This qualitative type of comparative dynamics has also been employed by Day (1994). His policy implications display a similar trade-off between stability and efficiency.

${ }^{3}$ As a referee pointed out, under an expectation-augmented price and wage adjustment scheme, there are only Walrasian steady states. In this case, however, a 2-parameter bifurcation shows that for no choice of policy parameters will such a state be stable. Hence, this main statement remains true.
} 


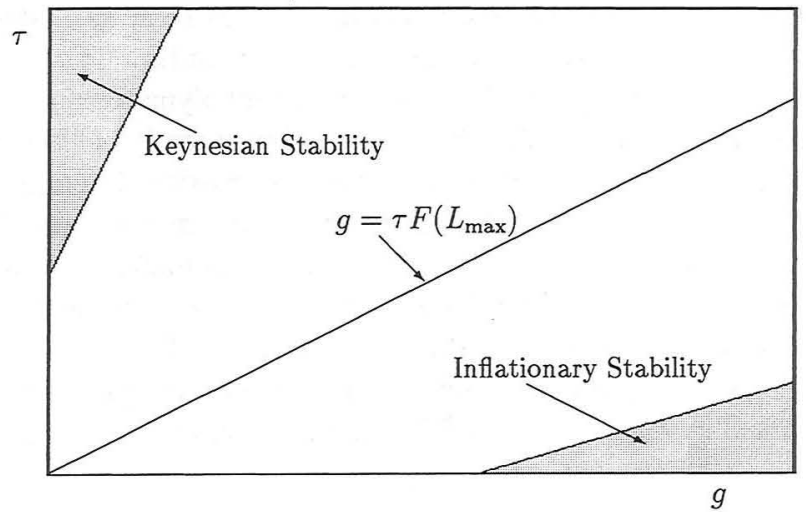

Fig. 1. Period doubling curves and stability regions (shaded).

$t+1$ its tax revenue of period $t$ (cf. Day (1994)). Thus, one has

$$
g_{t+1}=\frac{1}{1+\mathscr{P}\left(\alpha_{t}, m_{t} ; g_{t}, \tau\right)} \tau \mathscr{Y}\left(\alpha_{t}, m_{t} ; g_{t}, \tau\right),
$$

so that government expenditures are an endogenous variable and the tax rate is the sole fiscal policy instrument. One can easily show that only Walrasian steady states exist

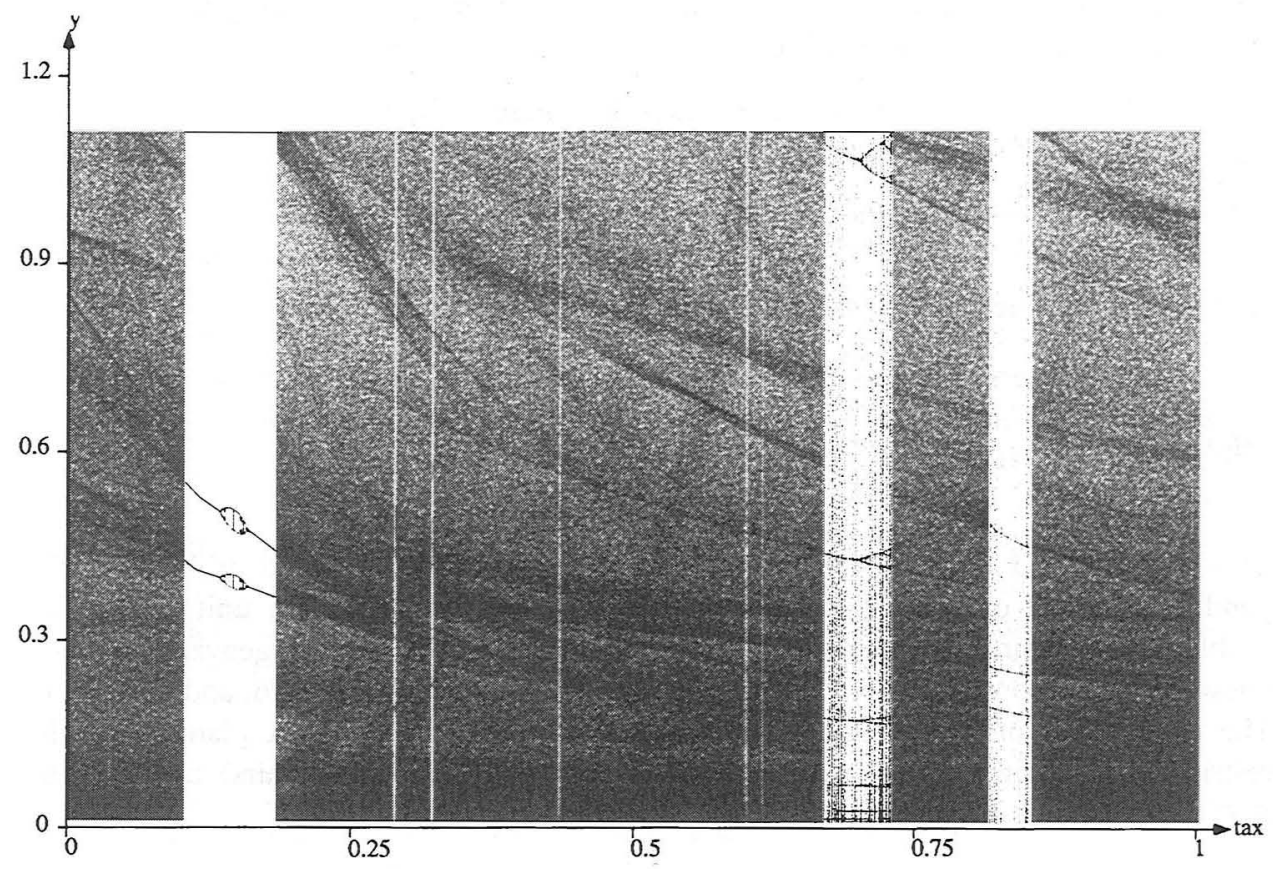

Fig. 2. Bifurcation diagram for real income and tax rate changes for a balanced budget policy. 
under such a policy, and one might expect that at least for some tax rates such a state is stable. To check this numerically, we fix all parameters as in Eq. (1) and the initial values $\left(\alpha_{o}, m_{o}, g_{o}\right)=(1,4,0.5)$, and compute 2000 iterations of the dynamical system for different tax rates between 0 and 1 . Since only the long run behavior is of interest, the first 200 iterations are eliminated. The result is shown in the bifurcation diagram of Fig. 2, where the dynamics of the real income is plotted against different tax rates. It turns out that irregular or periodic fluctuations result for all tax rates so that this policy rule does not stabilize an efficient steady state. Surprisingly, except for periodic windows the range of incomes seems not to be affected by different tax rates. In order to find stabilizing policies, it thus seems reasonable to follow the approach of applying the control methods for chaotic dynamical systems introduced in the next section.

\section{The methods of controlling chaos}

\subsection{The locul control method}

Consider a nonlinear discrete-time dynamical system

$$
x_{t+1}=\mathscr{F}\left(x_{t}, q\right),
$$

where we assume that $x_{t} \in \mathbb{R}^{n}$ is the state variable and $q \in \mathbb{R}$ is the only available control parameter which we allow to vary in the interval $|q-\bar{q}| \leq \Delta q$. We assume further that we are in the neighborhood of some steady state $(\bar{x}, \bar{q})$, which we want to stabilize by choosing an appropriate sequence of admissible parameters $q_{t}$. Linearizing the map $\mathscr{F}$ in $(\bar{x}, \bar{q})$ yields the linear control system

$$
x_{t+1}-\bar{x} \simeq A\left(x_{t}-\bar{x}\right)+B\left(q_{t}-\bar{q}\right),
$$

where

$$
\Lambda=d_{x} \mathscr{F}(\bar{x}, \bar{q}) \in \mathbb{R}^{n, n} \text { and } B=d_{q} \mathscr{F}(\bar{x}, \bar{q}) \in \mathbb{R}^{n, 1} .
$$

If we assume a linear feedback rule for the parameter $q_{t}$ of the form

$$
q_{t}-\bar{q}=K\left(x_{t}-\bar{x}\right), \quad K \in \mathbb{R}^{1, n},
$$

Eq. (2) reads as

$$
x_{t+1}-\bar{x} \simeq(A+B K)\left(x_{t}-\bar{x}\right) .
$$

This shows that $\bar{x}$ will be stabilized for the linearized equation and arbitrary initial conditions if and only if all the eigenvalues of $A+B K$ lie inside the unit circle. The problem of choosing the matrix $K$ such that $A+B K$ takes on given eigenvalues is well known in linear control theory (see Kwakernaak and Sivan, 1972, Th.6.6. and Th.6.26.)): The eigenvalues of $A+B K$ can be arbitrarily located in the complex plane (with the restriction that complex eigenvalues occur in complex conjugate pairs) by choosing $K \in \mathbb{R}^{1, n}$ suitable, if and only if the matrix

$$
C:=\left(B|A B| A^{2} B|\ldots| A^{n-1} B\right) \in \mathbb{R}^{n, n}
$$


is of rank $n$. Note that this controllability condition is satisfied for almost all pairs of matrices $(A, B) \in \mathbb{R}^{n, n} \times \mathbb{R}^{n, 1}$.

Since $\mathscr{F}$ is assumed to be nonlinear, the application of linear control theory will succeed only in a sufficiently small neighborhood $U$ around $\bar{x}$. Such a neighborhood can be expected to be entered if the system has a natural invariant measure $\mu$ with $\mu(U)>0 .{ }^{4}$ Furthermore, taking into account the maximal allowed deviation from the nominal parameter $\bar{q}$, we are restricted to the strip $S_{K}$ defined by

$$
S_{K}=\left\{x \in \mathbb{R}^{n}|| K(x-\bar{x}) \mid \leq \triangle q\right\} .
$$

Clearly, since any choice of the eigenvalues of $A+B K$ inside the unit circle satisfies our purpose, there are many possible choices of the matrix $K$. In particular, it seems to be reasonable to choose all the desired eigenvalues equal to zero. This would imply $(A+B K)^{n}=0$, and in a linear system the target would be reached at least after $n$ periods. However, since we are in a nonlinear setting, this choice need not be appropriate, because the neighborhood of convergence may be very small.

Romeiras et al. (1992) investigated numerically which choice of the feedback matrix $K$ exhibits the shortest stabilization time. In the two-dimensional case, if $\bar{x}$ is a saddle point, then the optimal $K$ is such that $A+B K$ projects onto the linearization of the stable manifold. This leads to the control formula originally developed by Ott et al. (1990). The matrix $K$ can be computed as follows: Take one left eigenvector $\nu$ of the unstable eigenvalue $\lambda_{u}$, that is, $\nu \cdot A=\lambda_{u} \cdot \nu$. Since $\nu$ is orthogonal to the stable eigenvector of $A$, the claim that $A+B K$ projects onto the stable manifold is equivalent to

$$
0=\nu(A+B K)=\lambda_{u} \cdot \nu+\nu B K .
$$

This yields $K=-\lambda_{u}(\nu B)^{-1} \nu$ and hence the control formula

$$
q_{t}=q-\frac{\lambda_{u} \nu\left(x_{t}-\bar{x}\right)}{\nu B} .
$$

The strip $S_{K}$ is in this case a vicinity of width $\triangle q /|K|$ of the linear stable manifold. The local control algorithm will be activated as soon as the trajectory will meet the set $S_{K}$ for the first time. Note, however, that because of nonlinearities it is not guaranteed that this process will converge. It can happen that the trajectory will leave the strip again and continue to move irregularly. From this point of view it seems to be better to activate the control only in the ball of radius $\triangle q /|K|$ with center $\bar{x}$, but this choice usually has the disadvantage that the time until the first control is activated will take much longer. $A$ similar control formula for the higher dimensional case and also for the stabilization of higher periodic orbits has been elaborated by Romeiras et al. (1992).

Assume that we want to activate the local control in a ball of radius $r=\Delta q /|K|$ around $\bar{x}$, denoted by $\mathscr{B}_{r}$ If the dynamical system has natural invariant measure $\mu$, the expected time $\tau$ required to reach $\mathscr{B}_{r}$ is equal to $1 / \mu\left(\mathscr{B}_{r}\right){ }^{5}$ Since the natural measure

\footnotetext{
${ }^{4}$ The natural invariant measure $\mu(U)$ of a Borel set $U$ is, if it exists, defined as follows. Let $\chi_{U}$ denote the characteristic function of $U$. If $\lim _{t \rightarrow \infty} \frac{1}{t} \sum_{k=0}^{t-1} \chi U\left(\mathscr{F}^{k}(x)\right)$ is constant for almost all $x$ (w.r.t. the Lebesguemeasure), then this constant is called natural invariant measure of $U$ (see Farmer et al., 1983). Hence, $\mu(U)$ measures the average time a typical trajectory spends in $U$.

${ }^{5}$ This follows from Kac's Lemma, see Cornfeld et al. (1982)
} 
scales typically with the information dimension $D$ of the measure $\mu^{6}$, we have $\tau \simeq 1 / r^{D}$. This implies a power law dependence between the maximal parameter perturbation and the expected time to achieve control of the form $\tau \simeq c /(\Delta q)^{D}$. Hence there is a trade-off between the maximal allowed parameter changes (or the cost of control) and the expected time to achieve the target. Since $\Delta q$ may not be too large, there exists some lower limit of the expected time which may still be very large.

\subsection{The targeting method}

With the help of the targeting procedure, which is based on the sensitive dependence on small parameter changes, the time needed to reach such a neighborhood can be considerably shortened. In contrast to the local control method, this process is not a simple feedback control rule, but needs very extensive computations in advance.

In the following discussion a pure forward targeting method in distinction to the forward-backward method of Shinbrot et al. (1990) will be presented, since many dynamical systems (in particular, the one in Section 2) do not possess an inverse. Assume that we desire to reach the ball $\mathscr{B}_{r}$ and that we are starting from some point $x_{o}$. The set of points, which can be achieved in the next iteration, is in linear approximation

$$
\Lambda=\left\{\mathscr{F}\left(x_{o}, \bar{q}\right)+d_{q} \mathscr{F}\left(x_{o}, \bar{q}\right)(q-\bar{q})|q-\bar{q}| \leq \Delta q\right\} .
$$

The idea is to iterate this line segment with the nominal parameter $\bar{q}$, hoping that due to the stretching of the iterates we find an intersection of one iteration of $\Lambda$ with $\mathscr{B}_{r}$ Fig. 3 shows such a situation where all iterations of $\Lambda$ under $\mathscr{F}(., \bar{q})$ in state space, denoted $\mathscr{F}^{k}(\Lambda)$, are plotted. Then we determine the value of the parameter, viewed as a parameterization of $\Lambda$, that will be mapped into $\mathscr{B}_{r}$ and we choose this parameter as the first control step. Due to roundoff and linearization errors we have to correct the parameters in the next iterations according to the same scheme where we hope to need one iteration of the new set $\Lambda$ less than in the step before. In practice, we can only iterate

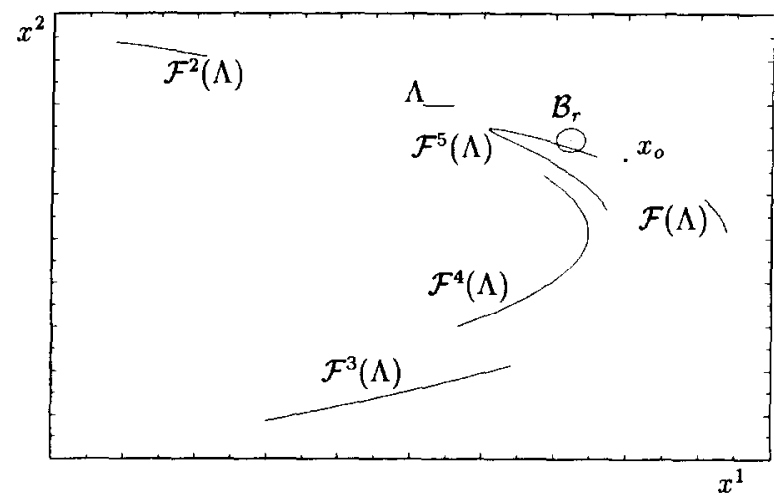

Fig. 3. The targeting procedure: the fifth iterate of the set $\Lambda$ intersects with the target region $\mathscr{R}_{r}$

\footnotetext{
${ }^{6}$ see Young (1982)
} 
discrete approximations of the set $\Lambda$. We will choose equally spaced points on the set $\Lambda$ and have to take care that their iterates do not deviate too much from each other.

An important point is the role of noise or modeling errors which was investigated by Ott et al. (1990) for the local control method and by Shinbrot et al. (1990) for the targeting procedure. They assumed that the 'real' dynamics satisfies $x_{t+1}=\mathscr{F}\left(x_{t}, q\right)+\kappa_{t}$ with some independently distributed random variables, $\kappa_{t}$, of small amplitude and found that the methods still remain effective provided that the noise is not too large. Improvements concerning the sensitivity to noise have been obtained by Dörner and Hübinger (1993). Furthermore, since the necessary informations about the dynamical system can be obtained from time series via delay coordinate embedding, it is possible to apply these methods without knowledge of the system's equations (see, e.g. Ditto et al., 1990).

\section{Stabilization of the Walrasian equilibrium}

We know from Proposition 2 that even in the unstable case a one-dimensional stable manifold exists. Considering a Walrasian equilibrium, where the system is nondifferentiable, we may nevertheless differentiate the equations in every one of the three disequilibria regimes and compute the stable eigenvectors. This raises the question, whether there exist parts of the stable manifolds (stable branches) which are invariant under the dynamics of the real system. By using the eigenvalues and eigenvectors computed for the proof of Proposition 2 one can prove that for large sets of parameters there exist such stable branches in the Keynesian and in the Inflationary regimes, but not in the Classical regime. But, as mentioned above, real wages fall in the Classical regime and so one can expect that Classical states very close to the Walrasian equilibrium will be mapped into one of the other regimes. The stable branches can be computed numerically and are shown by the solid lines in Fig. 4 for policy parameters

$$
g=0.2 \overline{7} \text { and } \tau=0.25 \text {. }
$$

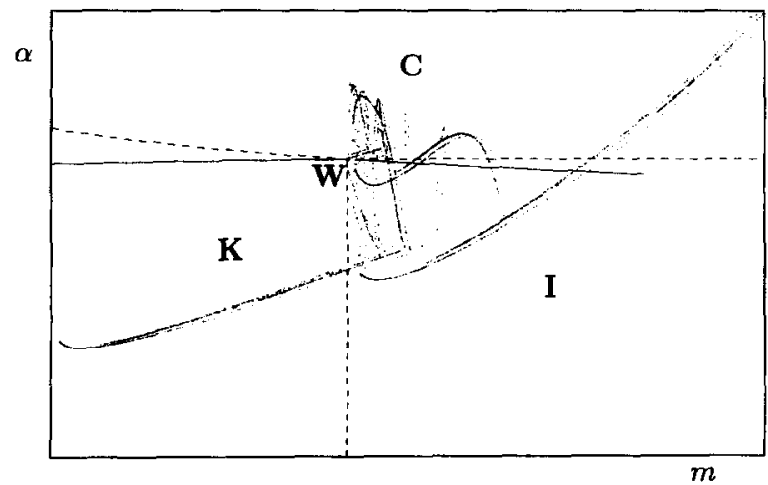

Fig. 4. Unstable Walrasian equilibrium with a strange attractor (1000 iterations), regime boundaries (dashed lines) and stable branches (solid lines). 
A strange attractor of $\mathscr{F}$ is plotted additionally, and the dashed lines indicate the boundaries between the three disequilibrium regimes.

The picture suggests that the Walrasian equilibrium is part of (or at least very close to) the attractor and that only the Inflationary branch intersects the attractor.

We will now apply the methods of Section 4 to the problem of stabilizing a stationary Walrasian equilibrium. Before we start our investigations, it should be remarked that it was not possible to prove a general result on the success of the local stabilization. This is due to the fact that the system is only piecewise differentiable and that the positions of the regime boundaries are also affected by variations of the policy parameters. For instance, it can happen that a state in $\mathbf{I}$, close to the boundary between $\mathbf{K}$ and $\mathbf{I}$, recommends a certain policy rule which displaces the boundary such that the state will be Keynesian and the policy ineffective. In spite of these difficulties, we will now concentrate on the problem of reaching the stable branches that we have found in two of the three disequilibrium regions. It will be shown that we still succeed with this procedure, even though no mathematical proof could be obtained.

First, fix nominal policy parameters $\bar{g}$ and $\bar{\tau}$ which satisfy a balanced government budget at full employment. The Walrasian steady-state is then given by

$$
\bar{\alpha}=F^{\prime}\left(L_{\max }\right) \text { and } \bar{m}=(1-\bar{c}(1-\bar{\tau})) F\left(L_{\max }\right)-\bar{g} .
$$

Denoting the general control parameter by $q$, we can think of four possible policies:

1. (Variation of government demand) $q=g$ and $\tau=\bar{\tau}$.

2. (Variation of the tax rate) $q=\tau$ and $g=\bar{g}$.

3. (Variation of $g$ and $\tau$ with balanced government budget) $q=\delta$ with $g=\delta \bar{g}$ and $\tau=\delta \bar{\tau}$.

4. Independent variation of $g$ and $\tau$.

Clearly, the fourth case is exceptional in the sense that we shall use a two-parameter control.

Consider for a moment the case of one control parameter. Denote the dynamical system by $x_{t+1}=\mathscr{F}\left(x_{t}, q_{t}\right)$ and the derivatives by

$$
A=d_{x} \mathscr{F}=\left(\begin{array}{ll}
a_{1} & a_{2} \\
a_{3} & a_{4}
\end{array}\right) \text { and } \quad B=d_{q} \mathscr{F}=\left(\begin{array}{l}
b_{1} \\
b_{2}
\end{array}\right) .
$$

(We omit indices for the different regimes.) Assuming the case with eigenvalues $\lambda_{u}, \lambda_{s}$ and $\left|\lambda_{u}\right|>1,\left|\lambda_{s}\right|<1$ we compute an unstable left eigenvector

$$
\nu=\left(\lambda_{u}-a_{4}, a_{2}\right),
$$

and rewrite formula Eq. (3) as

$$
q_{t}-\bar{q}=\frac{\lambda_{u}\left(a_{4}-\lambda_{u}\right)}{h}\left(\alpha_{t}-\bar{\alpha}\right)-\frac{\lambda_{u} a_{2}}{h}\left(m_{t}-\bar{m}\right)
$$

with

$$
h=\nu B=\left(\lambda_{u}-a_{4}\right) b_{1}+a_{2} b_{2} .
$$

We see that only the denominator $h$ in the above expression depends on the choice of the control parameter. Hence, in any case, the control parameter has to react to deviations 
in the real wage and the real balance in the same proportions. We should note that in both regimes $a_{4}=\partial m_{t+1} / \partial m_{t}$ is positive, and therefore $\lambda_{u}-a_{4}$ is negative.

Case 1. Variation of government demand

Government demand plays exactly the same role as real balances since both have the same influence on aggregate demand. This implies $b_{1}=a_{2}$ and $b_{2}=a_{4}$, and leads to the local control formula

$$
g_{t}-\bar{g}=-\frac{\lambda_{u}-a_{4}}{a_{2}}\left(\alpha_{t}-\bar{\alpha}\right)-\left(m_{t}-\bar{m}\right)
$$

On the one hand, $g$ has to react to deviations of $m$ from its target value $\bar{m}$ in the opposite direction, which indicates a clear counter-cyclical component in the policy rule. On the other hand, $g$ also has to react to deviations of the real wage from its full employment level. In $\mathbf{I}, a_{2}=\partial \alpha_{t+1} / \partial m_{t}<0$ holds true ${ }^{7}$ and so the coefficient is negative. Since real wages in $\mathbf{I}$ are always below their full employment level there is a positive effect on government demand, which either dampens the pure counter-cyclical response to the deviation in the real balance if real wages are relatively high (in Fig. 3, this means that the state is above the linear stable manifold), or exceeds it and implies a raise in government demand if real wages are relatively low. In the Keynesian regime the sign of $a_{2}$ is not uniquely determined. However, under the assumption of Proposition 3 (ii)), we have $a_{2}>0$ and a positive coefficient in front of $\alpha_{t}-\bar{\alpha}$. Thus, since for most Keynesian states close to the Walrasian equilibrium real wages are below their steadystate value, this will result in a dampening of the counter-cyclicity or a pro-cyclical reduction of government demand. Fig. 5 shows the time series of real balances and government demand for a typical stabilization sequence along the stable branch contained in $\mathbf{I}$.
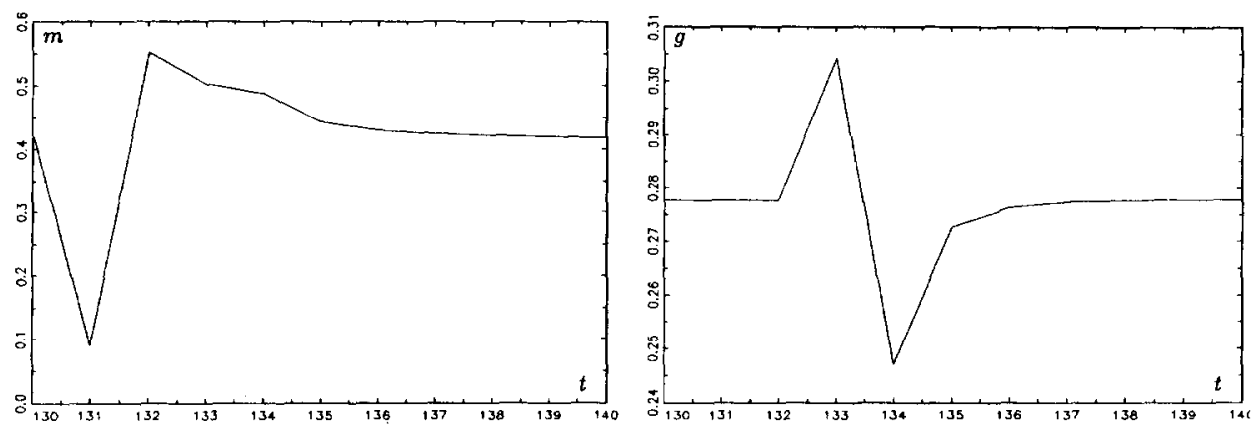

Fig. 5. Local control via government demand: Time series in $m$ and $g$.

\footnotetext{
${ }^{7}$ The argument is as follows: An increase in money balances increases excess demand for goods without affecting excess demand for labor. Thus, the goods price increases while the nominal wage remains constant, which implies a lower real wage in the next period.
} 
Case 2. Variation of income tax rates

Income tax rates influence aggregate demand, and also the savings of the young generation to the next period. Therefore, not only the pure demand effect has to be taken into account. We compute $b_{1}=-a_{2} \bar{c} F\left(L_{\max }\right)$ and $b_{2}=-\left(a_{4} \bar{c}+1\right) F\left(L_{\max }\right)$. This yields the denominator $h=-a_{2}\left(1+\bar{c} \lambda_{u}\right) F\left(L_{\text {max }}\right)$, which vanishes if $\bar{c} \lambda_{u}=-1$ holds true. In this case a pure tax rate policy will be ineffective. In order to compare the tax policy with the expenditure policy in Case 1 , we have to compare deviations $\triangle g$ with deviations $\triangle \tau F\left(L_{\max }\right)$. We find with the help of formula Eq. (4)

$$
\triangle \tau F\left(L_{\max }\right)=-\frac{\lambda_{u}}{1+\bar{c} \lambda_{u}} \triangle g
$$

and note that the coefficient has a modulus larger than 1. This implies that larger deviations in taxes than in government demand are required to place the trajectory onto the stable manifold. In other words, if we allow the government budget to vary in a certain range, then the neighborhood around the stable manifold, where the stabilization policy is activated, is much smaller for tax rate control than for expenditure control. Whether the tax policy has the same or different cyclicity properties as the expenditure policy depends on the sign of $1+\bar{c} \lambda_{u}$. However, in any case the amplitudes are higher for a stabilization through taxes. The lack of efficiency of the tax rate policy can be seen in Fig. 6. The stabilization takes place after four failures which are due to escapes of the trajectory into the Classical regime.

Case 3. Variation of $g$ and $\tau$ with balanced government budget

The control with a balanced government budget differs from the pure control via government demand (Case 1) about the factor $\lambda_{u} /\left(\lambda_{u}-\left(1+\bar{c} \lambda_{u}\right)\right)$ which is always positive and implies that this policy has the same cyclicity properties. In the case $\lambda_{u} \bar{c}=-1$, tax policy (Case 2) is ineffective, and so the expenditure policy coincides with the policy of Case 1. For $\bar{c} \lambda_{u}<-1$, which holds in our numerical examples, the coefficient is larger than one and cyclicity is amplified, while for $\bar{c} \lambda_{u}>-1$ it is dampened. According to this, in the first case the vicinity around the stable manifold where the local stabilization is activated, is smaller than in the second one and therefore the expected time to achieve such a neighborhood is longer.
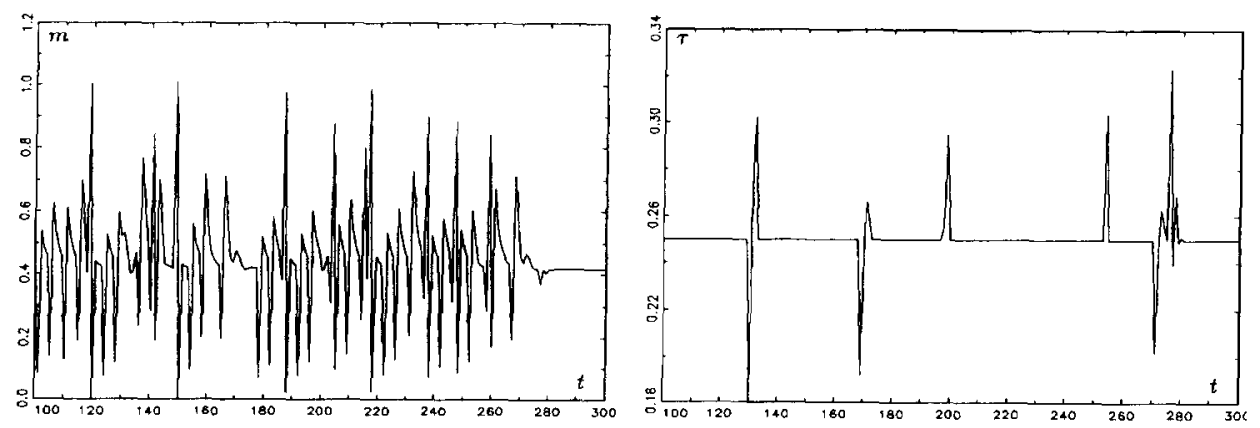

Fig. 6. Local control via tax rates: Time series in $m$ and $\tau$. 
Case 4. Independent control of government demand and tax rates

The independent control of government demand and tax rates has the advantage that we need not target the stable branches of the steady state, but may try to achieve the steady state directly. Formally, in a two-dimensional system $x_{t+1}=\mathscr{F}\left(x_{t} ; q_{1}, q_{2}\right)$ this means that we want to find feedback rules

$$
q_{1 t}-\vec{q}_{1}=K_{1}\left(x_{t}-\bar{x}\right) \text { and } q_{2 t}-\bar{q}_{2}=K_{2}\left(x_{t}-\bar{x}\right),
$$

such that

$$
A+B_{1} K_{1}+B_{2} K_{2}=0
$$

where $A=d_{x^{2}} \mathscr{F}, B_{1}=d_{q_{1}} \mathscr{F}$ and $B_{2}=d_{q_{2}} \mathscr{F}$. This is a four-dimensional linear equation in four unknowns $K_{1}$ and $K_{2}$, which can be solved uniquely if and only if $B_{1}$ and $B_{2}$ are linearly independent.

In particular, in our system we choose $q_{1}=g$ and $q_{2}=\tau$ and find that the derivatives with respect to $g$ and $\tau$ are linearly independent if and only if $a_{2}=\partial \alpha_{t+1} / \partial m_{t} \neq 0$. We compute the following local control formulas

$$
\begin{gathered}
g_{t}-\bar{g}=-\left(\frac{c}{a_{2}} \operatorname{det} A+\frac{a_{1}}{a_{2}}\right)\left(\alpha_{t}-\bar{\alpha}\right)-\left(m_{t}-\bar{m}\right) \\
\tau_{t}-\tau=-\frac{1}{F\left(L_{\max }\right) a_{2}} \operatorname{det} A\left(\alpha_{t}-\bar{\alpha}\right) .
\end{gathered}
$$

Hence, tax rates react only to deviations in the real wage, while government demand reacts to deviations in the real balance in the opposite direction corrected about a real wage term. This policy has the advantage that it can be applied in the Classical regime also and that it can be expected to be robust against small perturbations, because small deviations from the steady state will be placed back in a very short time.

In order to compare the stabilization time of different policies, we chose 1000 initial values randomly and iterated each of them for 1000 periods. For the first 100 periods we left the parameters constant. When a trajectory entered the neighborhood of the stable branch defined by a maximal deviation of the government budget about 0.1 (i.e. $\triangle g=0.1$ resp. $\Delta \tau=0.09$ ) for the first time, control was activated and the time retained. Additionally, we retained the first times of success of control which was defined by the entrance time in a small $\varepsilon$-neighborhood $(\varepsilon=0.0001)$ around the steady state. For all the four policies we counted the number of successful experiments, the average time of the first activation of control, and the average time of success of control. The results are plotted in Table 1.

Table 1

Efficiency of local control for different policies

\begin{tabular}{llll}
\hline Policy & Successful experiments & $\begin{array}{l}\text { Average first activation } \\
\text { of control }\end{array}$ & Average time of success \\
\hline 1 & 892 & 156.8 & 167.0 \\
2 & 646 & 232.4 & 518.4 \\
3 & 998 & 201.2 & 278.1 \\
4 & 995 & 182.7 & 269.1 \\
\hline
\end{tabular}



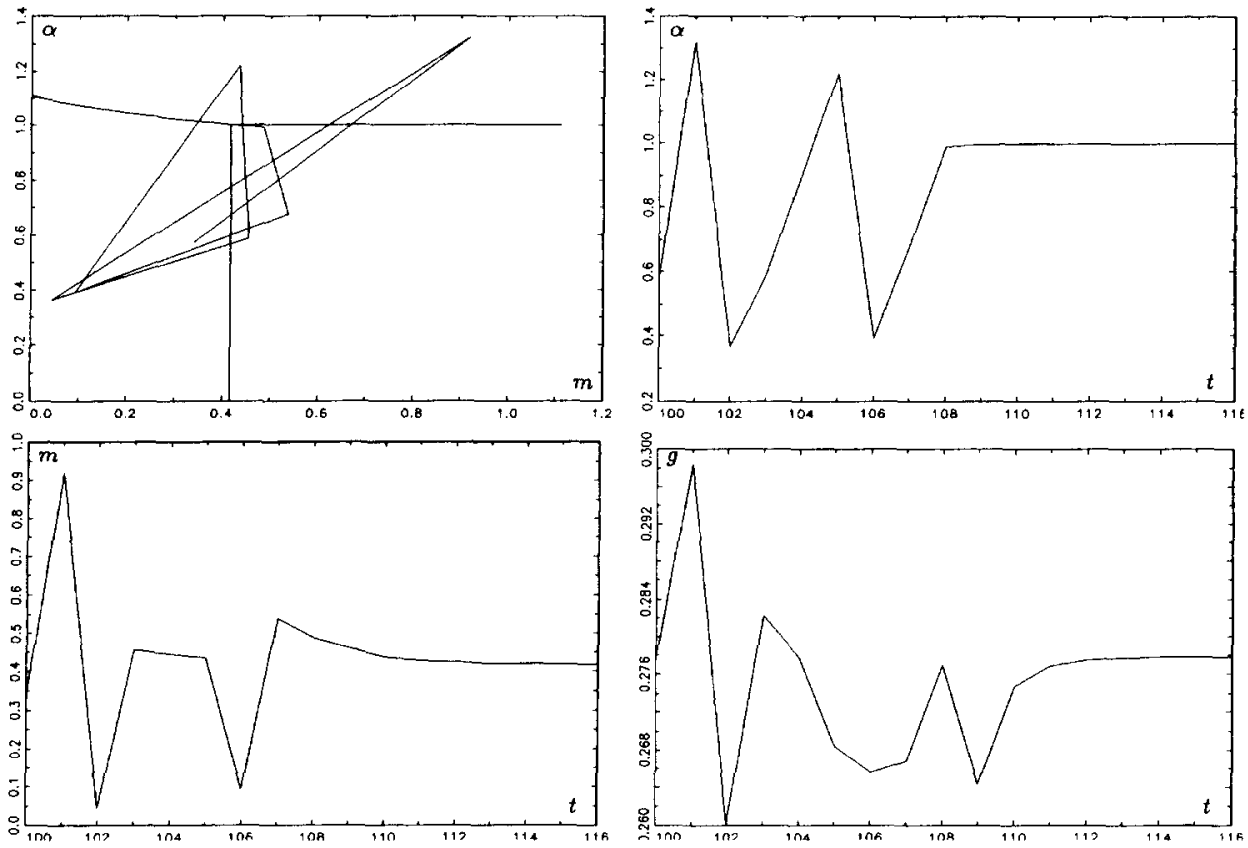

Fig. 7. A targeting path: Phase diagram and time series in $\alpha, m$ and $g$.

It turns out that the government demand policy is the best policy with respect to the average success time, while the pure tax policy displays the worst performance. However, even for such large variations of policy parameters, the expected time to succeed with the local control method can still be large. From an economic point of view, it is unsatisfying to wait 50 or more periods under renunciation of any policy measures until the economy settles down close to the equilibrium and a certain policy stabilizes it.

When applying the targeting method, it will usually lead to a neighborhood of the equilibrium in a short time without any time for free running in the beginning. In our experiments we applied a combination of the targeting and the local control method. Numerically the strip $\Lambda$ (cf. Section 4 ) was approximated by 50 equally spaced points which were iterated as long as the connecting lines between these iterated points intersected with one of the linearized stable branches for the first time. The corresponding parameter was chosen as the first targeting step, and the local control was switched on as soon as the trajectory settled down in the vicinity $S_{K}$ (cf. Section 4) around the stable branch. Fig. 7 shows a targeting path and the corresponding time series of the system variables and government demand.

The gain of efficiency of the targeting algorithm is indicated in Table 2. Here we started with 25 randomly distributed initial values, iterated them 100 times without control and retained the first times when the targeting algorithm and the local control was switched on and when the control was successful. No significant differences between the different policies can be observed (Case 4 was neglected since we do not know of a targeting algorithm for two system parameters). 
Table 2

Efficiency of targeting for different policies

\begin{tabular}{llll}
\hline Policy & Average first targeting step & Average first local control step & Average time of success \\
\hline 1 & 101.1 & 109.8 & 117.2 \\
2 & 101.9 & 110.0 & 115.2 \\
3 & 101.1 & 106.2 & 116.9 \\
\hline
\end{tabular}
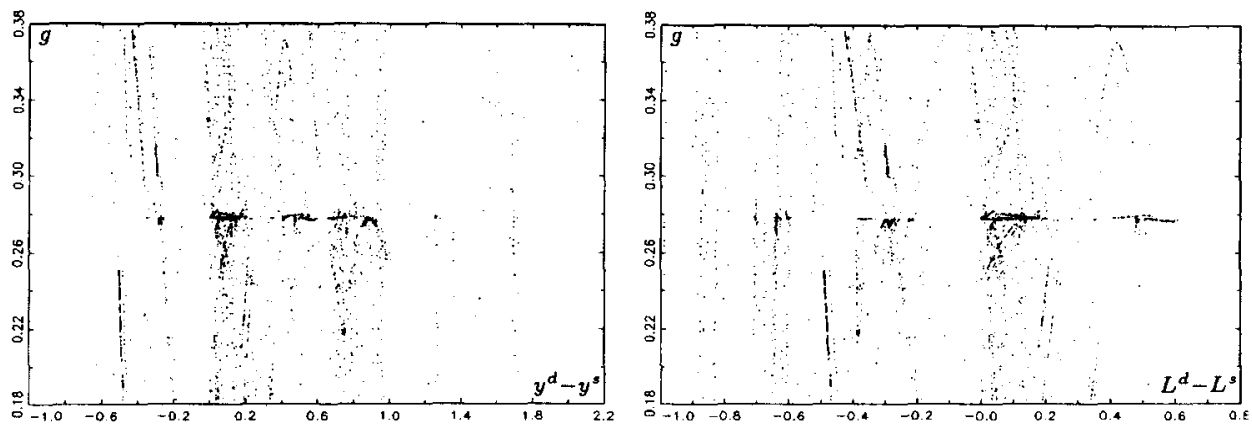

Fig. 8. 3600 targeting steps in $g$ vs. excess demand for goods and labor.

Up to now the application of the targeting method needs global knowledge of the underlying dynamical system or at least a good enough approximation obtained from long enough time series. Both claims are, of course, too strong and not very plausible from a behaviorist point of view. Therefore, it is necessary to study whether the policies obtained from the targeting algorithm exhibit correlations with observable market signals. If this was the case, successful policy rules might be obtained which use such limited informations only.

Fig. 8 shows, however, that this is not the case. Here, 3600 targeting policies compared to the disequilibria on the goods market and on the labor market are plotted. The picture shows that there is no clear dependence between the policies and the 'observable' market signals. Thus, it seems impossible for a central planner to find the right policy solely by the knowledge of actual disequilibrium signals. This can be explained by the argument that a complicated dynamics should not stabilizable globally with a simple policy. It is clear that due to the sensitive dependence on initial conditions two different states with economically meaningless difference will distinguish from each other dramatically after a few iterates and therefore require quite different policy interventions in the beginning which cannot be recognized by the government.

In case of a periodic attractor, it is also possible to stabilize a stationary equilibrium, provided the system exhibits transient chaotic behavior. Tél (1993) investigated such a system and found that an application of the local control method implies that the maximal allowed perturbation of the parameter is proportional to the fraction of initial conditions which will achieve the neighborhood around the equilibrium where local control is applicable. A better performance, however, can be achieved if we also apply the targeting 

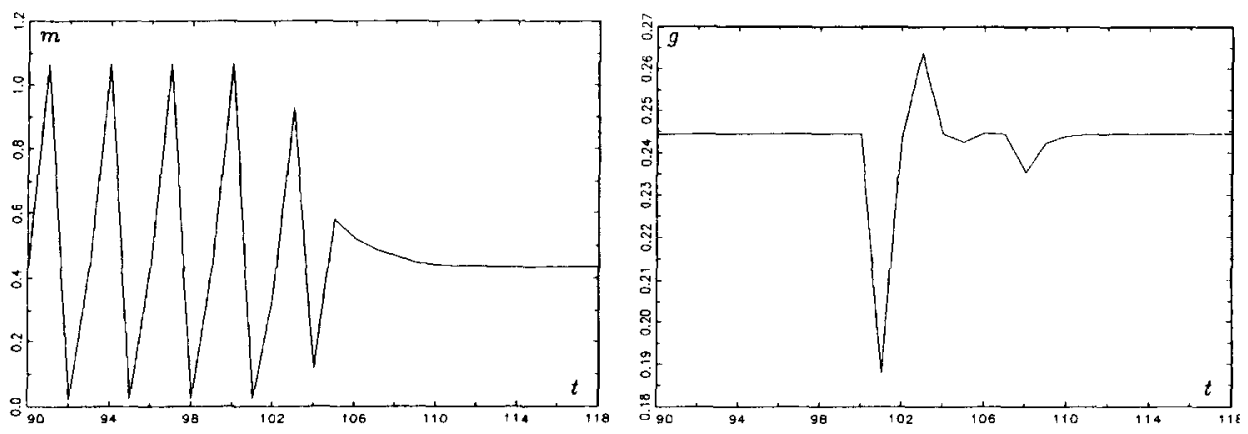

Fig. 9. Destabilization of a 3-cycle and stabilization of W: Time series in $m$ and $g$.

method. Even more, if the initial perturbation chosen is large enough, we are able to destabilize the periodic cycle and to target a Walrasian equilibrium outside of the attracting set. Fig. 9 shows such a destabilization of a period-3 cycle and the stabilization of the Walrasian equilibrium with a variation of $\triangle g=0.1$ about its mean value $\bar{g}=0.2 \overline{4}$ and constant tax rate $\bar{\tau}=0.22$. This indicates that these methods, not only allow avoidance of irregular behavior, but also avoid cyclicity, provided the dynamics outside of the cycle is flexible enough.

\section{Conclusion}

The methods of Controlling Chaos provide a useful tool for the stabilization of nonlinear dynamical systems. By varying parameters in a certain range, undesired irregular or even cyclical behavior can be avoided. The method of local control requires information on the steady-state position only and the first derivative of the dynamical equations at the steady state, but can exhibit undesirable long transients. The policy rule depends piecewise linearly on deviations of the steady state and can be explained very well. In contrast to this, with the help of the targeting method, the steady state can be stabilized in a short time, but complete informations on the global dynamics are required. Moreover, there seems to be no simple adaptive policy rule generating these policies and a complete knowledge of the dynamics is essential to the application of this method.

Recently, other applications of these methods in economic contexts were given: Holyst et al. (1996) applied the Ott-Grebogi-Yorke method to a model of two competing firms, and Kopel (1997) showed in a competitive market model how firms can improve their performance measures by use of the targeting method. All these approaches, however, are based on descriptive modeling. As is well known, in dynamic optimization models also the optimal policy function may display chaotic behavior (see, e.g. Boldrin and Montrucchio, 1986). In this case, the decision maker prefers the chaotic policy to any regular policy. Hence, there is no need for avoiding the irregularities, unless other agents with different objectives are introduced. 
The significance for economic theory is the fact that many deterministic dynamical systems, even though they exhibit very irregular behavior, are in fact stabilizable, quite in contrast to systems with irregularities depending solely on stochastic shocks. However, as long as the complete dynamics is not well known to the central planner, the performance of a global stabilization policy is as much a question of incident as in a completely stochastic setting. On the other hand, a local stabilization can be achieved by simple adaptive policy rules depending on the state variables of the dynamical system. However, it is not even clear whether the government can observe all state variables or has knowledge only of certain observable market signals. In such a case one has to find out whether stabilization can be obtained by adaptive policy rules depending only on observable variables.

\section{Acknowledgements}

This research is part of the project Dynamische Makroökonomik supported by the DFG. I would like to thank R. Day, M. Kopel, and two anonymous referees for many valuable comments.

\section{References}

Benassy, J.-P., 1975. Neo-Keynesian disequilibrium theory in a monetary economy, Review of Economic Studies $42,502-523$.

Böhm, V., 1993. Recurrence in Keynesian macroeconomic models. In: Gori, F., Geronazzo, L., Galeotti, M. (Eds.), Nonlinear Dynamics in Economics and the Social Sciences, Springer-Verlag, Berlin, pp. 69 94.

Böhm, V., Lohmann, M., Lorenz, H.-W., 1994. Dynamic complexity in a Keynesian macro-model, Discussion Paper No. 288, University of Bielefeld.

Boldrin, M., Montrucchio, L., 1986. On the indeterminacy of capital accumulation paths, Journal of Economic Theory 40, 26-39.

Clower, R.W., 1965. The Keynesian counter-revolution: A theoretical appraisal. In: Hahn, F.M., Brechling, F.P. (Eds.), The Theory of Interest Rates, Macmillan, London, pp. 103-125.

Cornfeld, I.P., Fomin, S.V., Sinai, Y.G., 1982. Ergodic Theory, Springer-Verlag, New York.

Day, R.H., 1994. Business cycles, Fiscal policy and budget deficits, chapter 4 in Semmler, W. (Ed.), Business Cycles: Theory and Empirical Methods, Kluwer Publishing Co., New York, 113-143.

Ditto, W.L., Rauseo, S.N., Spano, M.L., 1990. Experimental control of chaos, Physical Review Letters 65 , 3211-3214.

Dörner, R.H., Hübinger, B., 1993. Local control of chaotic motion, Zeitschrift für Physik B 90, $103-106$.

Farmer, J.D., Ott, E., Yorke, J.A., 1983. The dimension of chaotic attractors, Physica 7D, 153-180.

Holyst, J.A., Hagel, T., Haag, G., Weidlich, W., 1996. How to control a chaotic economy?, Journal of Evolutionary Economics 6, 31-42.

Kaas, L., 1995. Steady states and local bifurcations in a dynamic disequilibrium model, Discussion Paper No. 300, University of Bielefeld.

Kopel, M., 1997. Improving the performance of an economic system: Controlling chaos, Journal of Evolutionary Ecunumics 7, 269-289.

Kwakernaak, H., Sivan, R., 1972. Linear Optimal Control Systems, John Wiley, New York.

Malinvaud, E., 1977. The Theory of Unemployment Reconsidered, Basil Blackwell, Oxford.

Ott, E., Grebogi, C., Yorke, J.A., 1990. Controlling chans, Physical Review Letters 64, 1196-1198. 
Phillips, A.W., 1954. Stabilization policy in a closed economy, Economic Journal 64, 290-323.

Romeiras, F.J., Grebogi, C., Ott, E., Dayawansa, W.P., 1992. Controlling chaotic dynamical systems, Physica D $58,165-192$.

Shinbrot, T., Ott, E., Grebogi, C., Yorke, J.A., 1990. Using chaos to direct trajectories to targets, Physical Review Letters 65, 3215-3218.

Tél, T., 1993. Crossover between the control of permanent and transient chaos, International Journal of Bifurcation and Chaos 3, 757-764.

Young, L.-S., 1982. Dimension, entropy and Liapunov exponents, Ergodic Theory and Dynamical Systems 2, 109-124. 